\title{
A HUMANIZAÇÃO NO ESCOPO DA FORMAÇÃO ACADÊMICA DOS GRADUANDOS EM ODONTOLOGIA, ENFERMAGEM E MEDICINA À LUZ DOS PROJETOS POLÍTICO-PEDAGÓGICOS
}

\author{
HUMANIZATION INTHE SCOPE OF ACADEMIC TRAINING OF UNDERGRADUATE STUDENTS IN \\ DENTISTRY, NURSING AND MEDICINE INTHE LIGHT OF PEDAGOGICAL POLITICAL PROJECTS
}

\begin{abstract}
Rita de Cássia Martins Moraes', Camila Moraes Albuquerque2, Marcos Antônio Albuquerque de Senna ${ }^{3}$, Henrique Eduardo Oliveira ${ }^{4}$, Karinne Bueno Antunes ${ }^{5}$, Larissa Maria Assad Cavalcante ${ }^{6,7}$
\end{abstract}

\section{Resumo}

Um dos maiores desafios na formação acadêmica do profissional de saúde é a conscientização da importância da humanização no atendimento, que, além de valorizar o cuidado em suas dimensões técnicas e científicas, reconhece também o direito dos pacientes, sua subje-tividade, individualidade e autonomia. Este trabalho objetivou identificar e analisar, por meio de uma pesquisa documental, a presença do termo humanização nos Projetos Político-Pedagógicos e Diretrizes Curriculares dos cursos de: Odontologia, Enfermagem e Medicina da Universidade Federal Fluminense em Niterói, no estado do Rio de Janeiro. Foi realizada uma pesquisa exploratória descritiva, com abordagem qualitativa. A análise documental dos currículos evidenciou uma aproximação com o tema nos três cursos estudados, porém com variações importantes no que se refere ao método de aplicação dos conteúdos teórico-práticos, ao longo do processo de formação. Nessa perspectiva, o curso de Medicina apresentou uma distribuição mais homogênea dos conteúdos relacionados ao termo humanização encontrado nas suas disciplinas obrigatórias. Foi possível, também, perceber o empenho dos cursos em contemplar as Novas Diretrizes Curriculares tendo a humanização como elemento prioritário no que se refere à construção de novas relações entre alunos, docentes, usuários dos serviços e a rede do Sistema Único de Saúde (SUS) local. Conclui-se que os três cursos estudados apresentam uma aproximação com o tema, esforçam-se em incorporar os pressupostos da humanização, tais como: ética, respeito, acolhimento, e procuram uma maior aproximação entre os sujeitos envolvidos na construção de novas relações entre alunos, docentes e usuários dos serviços e a rede SUS local.

Palavras - chave: Humanização; Medicina; Odontologia; Enfermagem; Formação Acadêmica.

\begin{abstract}
One of the greatest challenges in the health professional training is to be aware of the importance of humanization in care, in which, in addition to valuing care in its technical and scientific dimensions, they also recognize patients' rights, their subjectivity, individuality and autonomy. This work aimed to identify and analyze, through documentary research, the presence of the term humanization in the Political Pedagogical Projects and Curricular Guidelines of the Medicine, Dentistry and Nursing courses of the Federal Fluminense University. A descriptive exploratory research was carried out with a qualitative approach. The documentary analysis showed an approximation with the theme in the three courses studied, but with important variations regarding the method of application of the theoretical-practical content, throughout the training process. In this perspective, the Medicine course presented a more homogeneous distribution of the contents related to the term humanization found in its obligatory subjects. It was also possible to perceive the commitment of the courses to contemplate the New Curricular Guidelines, with humanization as a priority element in the construction of new relationships among students, teachers, users of the services and the local Public Health System (Sistema Único de Saúde - SUS). It is concluded that the three courses studied present an approxima-tion with the theme, strive to incorporate the presuppositions of humanization, such as: ethics, respect, acceptance, and seek a closer approximation between the subjects involved in the construction of new relations among students, teachers and users of the services and the local SUS.
\end{abstract}

Keywords: Humanization; Dentistry; Nursing; Medicine; Teaching

\footnotetext{
I. Professora Associada do Departamento de Odontotécnica, Faculdade de Odontologia, Universidade Federal Fluminense - UFF Niterói - RJ 2. Mestre em Odontologia, Área de Clínica Odontológica, Faculdade de Odontologia, Universidade Federal Fluminense - UFF Niterói - RJ

3. Professor Associado do Instituto de Saúde Coletiva com exercício no Departamento de Saúde e Sociedade, Faculdade de Odontologia, Universidade Federal Fluminense - UFF Niterói - RJ

4. Professor Associado do curso de graduação em Odontologia, Faculdade de Odontologia, Universidade Federal Fluminense - UFF Niterói - RJ

5. Doutoranda no Curso de Doutorado da Universidade Estadual do Rio de Janeiro - UERJ - Rj

6. Professora Associada do Departamento de Odontologia Faculdade de Odontologia, Universidade Federal Fluminense - UFF Niterói - RJ.

7. Professora do Programa de Pós-graduação em Odontologia, Faculdade de Odontologia, Universidade Veiga de Almeida - UVA Rio de Janeiro - RJ.
}

Como citar este artigo:

Moraes RCM, Albuquerque CM, Senna MAA, Oliveira HE, Antunes KB, Cavalcante LMA. A humanização no escopo da formação acadêmica dos graduandos em Odontologia, Enfermagem e Medicina à luz dos projetos político-pedagógicos. Rev Nav Odontol. 2021; 48 (2): . I 7-25. 


\section{INTRODUÇÃO}

Um dos maiores problemas encontrados na preparação do profissional de saúde é a pouca importância dada à humanização na formação acadêmica, possivelmente pela visão mecanicista e reducionista do homem, uma vez que ele não é visto como um todo, mas como um conjunto de partes tratadas separadamente perdendo a noção da unidade do todo biológico. Reconhecer apenas as necessidades biológicas e fisiológicas seria insuficiente para visualizar o ser humano como um todo. Desde 1970, a sociologia médica norte-americana tem concentrado esforços para conceituar o termo humanização e desumanização no cuidado em saúde (I).

Enriquecendo o significado de humanismo, Rios (2009) aborda o termo sobre várias concepções (2). No sentido filosófico, a humanização encontra suas raízes no Humanismo, corrente filosófica que procura entender o homem e indicar meios para que os indivíduos compreendam uns aos outros, entretanto sua leitura psicanalítica se volta à posição da subjetividade no campo da saúde. A humanização se caracteriza por tornar humano, ou seja, é a admissão de todas as dimensões humanas — históricas, sociais, artísticas, subjetivas, sa-gradas ou nefastas -, possibilitando escolhas conscientes e responsáveis. Além disso, a relação humanizada entre médico e paciente vem sendo abordada na literatura da antropologia médica, corroborando a necessidade desses pressupostos na formação do médico e dos demais profissionais de saúde.

$\mathrm{Na}$ abordagem do termo humanização, parece consenso que a questão central é o sujeito, a pessoa que busca pelo serviço de saúde, caracterizando, assim, uma assistência humanizada, personalizada (3).

No ano 2000, o Ministério da Saúde criou - Programa Nacional de Humanização da Assistência Hospitalar (PNHAH) visando a estimular a disseminação das ideias da humanização, os diagnósticos situacionais e a promoção de ações humanizadoras, tendo em vista a realidade local. Em 2003, o $\mathrm{PNHAH}$ passou por uma revisão e o Ministério da Saúde lançou a Política Nacional de Humanização (PNH), "que mudou o patamar de alcance da humanização dos hospitais para toda a rede SUS e definiu uma política cujo foco passou a ser, principalmente, os processos de gestão e de trabalho" (2). Com essa política, a humanização acessa processos de grande importância, tais como a gestão e a organização do trabalho nos serviços de saúde, despontando a gestão participativa como modelo eleito para a realização dela. A $\mathrm{PNH}$ adota a humanização como sendo um conjunto de princípios e diretrizes que são tra-duzidos em ações nos distintos serviços, práticas de saúde e instâncias do sistema, com características de uma construção coletiva. A Política Nacional de Humanização apresenta uma proposta de humanização vista não como um programa, mas como uma política que atravessa as diferentes ações e instâncias gestoras do SUS.

No que se refere ao processo de formação na saúde, a Lei de Diretrizes e Bases da Educação Nacional, Lei 9.394 de dezembro de 1996, assegura ao Ensino Superior maior flexibilidade na organização curricular dos cursos e se revela incongruente com as tendências contemporâneas de considerar a formação no âmbito da graduação como uma etapa inicial da formação continuada; bem como a crescente heterogeneidade tanto da formação prévia como das expectativas e dos interesses dos alunos (4).

Com as Diretrizes Curriculares Nacionais (DCN), demandas foram criadas para as Instituições de Ensino Superior (IES), relacionadas à qualidade do ensino de graduação e impondo outros desafios para os gestores dos cursos, para a formação e a prática dos docentes, já que não se restringem apenas à revisão dos conteúdos curriculares. Assim, novos Projetos Pedagógicos fazem-se essenciais para a formação dos novos profissionais de saúde.

$\bigcirc$ Projeto Político-Pedagógico (PPP) se constitui em uma ação intencional, com um significado claro e um compromisso escolhido de forma coletiva em busca de um rumo, de uma direção (5).

A humanização da atenção em saúde tem sido amplamente difundida, explorada e propagada. Não se pode negar sua importância e necessidade mediante um mundo globalizado, no qual o paciente chega com informações 
e questionamentos, que se traduzem em níveis de expectativas em relação ao atendimento e também com a finalização do trabalho clínico proposto e aceito por ele. Portanto, torna-se relevante a forma de abordagem do termo na formação dos profissionais em saúde e não apenas uma simples reprodução literal dos PPP, sendo indispensável a compreensão por parte dos alunos dos diversos significados e formas de aplicação da palavra "humanização".

No presente estudo, o termo humanização é conceituado em atitudes que, além de valorizarem o cuidado em suas dimensões técnicas e científicas, reconhecem também o direito dos pacientes, sua subjetividade, individualidade e autonomia, isto é, o encontro de sujeitos no, e, pelo ato de cuidar, o encontro das subjetividades. Ressalta-se que essa definição está coerente com a do Ministério da Saúde (6).

Esta pesquisa teve como objetivo identificar e analisar através de seus PPP a presença do termo humanização na formação acadêmica em saúde dos cursos de Medicina, Enfermagem e Odontologia de uma Instituição Pública de Ensino Superior, buscando em sua grade curricular as disciplinas obrigatórias comprometidas com o termo.

\section{MÉTODOS}

\section{Tipo de pesquisa e amostra}

Trata-se de um estudo quanti-qualitativo de caráter descritivo e exploratório, uma vez que se procurou entender, através de documentos públicos institucionais, como o termo humanização é trabalhado ao longo da formação dos alunos de graduação dos cursos de Medicina, Enfermagem e Odontologia de uma Instituição Pública de Ensino Superior. Foi realizada uma revisão bibliográfica e análise documental dos Projetos Político-Pedagógicos (PPP) da instituição. Esses cursos foram escolhidos por serem os mais antigos e por terem passado por diversos processos de mudanças e revisão dos seus PPP — exceto a Odontologia, que iniciou o seu processo de mudança mais recentemente.

A análise documental visou a identificar em documentos primários de cada curso os PPP e informações que pudessem servir de dados para nortear a análise em questão (7).

\section{Coleta de dados}

De cada um dos três cursos de graduação da área de saúde, utilizou-se a coleta de dados dos PPP formulados na sua implantação. Foram selecionados, das disciplinas obrigatórias, o termo humanização ou termos afins como: cuidar, humanizar, acolhimento, respeito e ética.

$\bigcirc$ método em questão tem como objetivo identificar em documentos primários informações que sirvam de subsídio para esclarecer alguma dúvida da pesquisa. Por ser uma fonte natural de informação, documentos não são considerados apenas a origem de informação contextualizada, mas surgem de um determinado contexto e fornecem informações sobre esse mesmo contexto (8).

\section{Tratamento dos dados}

Os dados coletados, considerando presentes os termos já citados pelo menos uma vez nas ementas e objetivos de cada disciplina obrigatória nos PPP de cada curso em estudo, foram identificados, estratificados, quantificados e apresentados na forma de gráficos relativos (percentuais) e tabelas.

\section{RESULTADOS}

A investigação relativa ao Percentual de Carga Horária Total das disciplinas obrigatórias de cada curso que cita o termo estudado e correlatos está apresentada no Gráfico I.

O Gráfico 2 demonstra a presença dos termos e seus correlatos, em disciplinas obrigatórias dos cursos estudados, analisando a carga horária teórica ou prática. Observou-se que a maior incidência dos referidos termos encontra-se nas disciplinas teóricas. $\bigcirc$ curso de Enfermagem é o que demonstra um maior equilíbrio entre a teoria e a prática e o de Odontologia é o que mais se distancia.

No curso de Medicina, que tem sua carga horária total de 9.270h, contando com 5.660 hora/aulas de disciplinas obrigatórias, consideradas no estudo, e dessas, apenas 3.456 hora/ aulas trataram do tema, conforme descreve a 


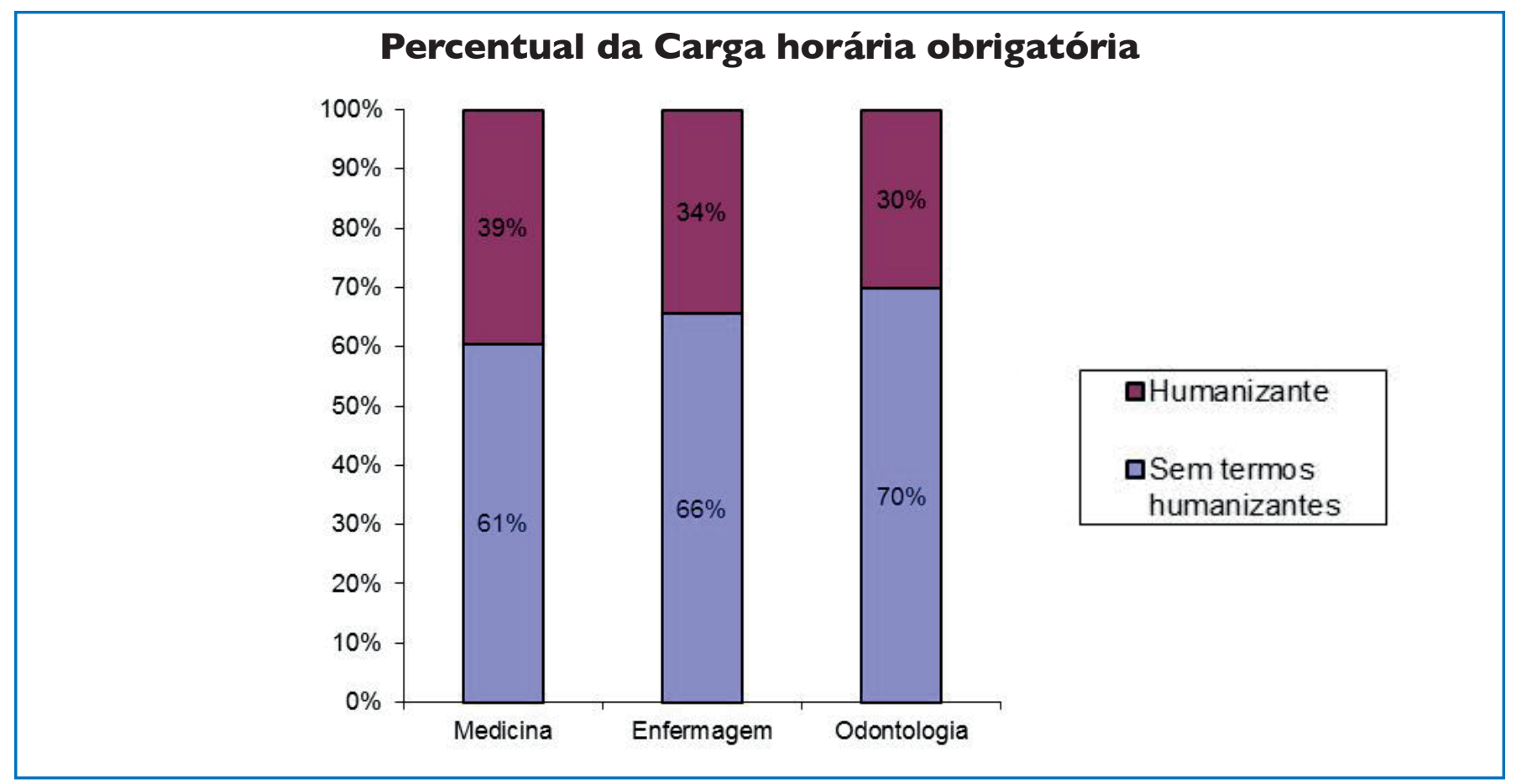

Gráfico I - Percentual de carga horária das disciplinas obrigatórias que utilizam termos humanizantes ou correlatos.

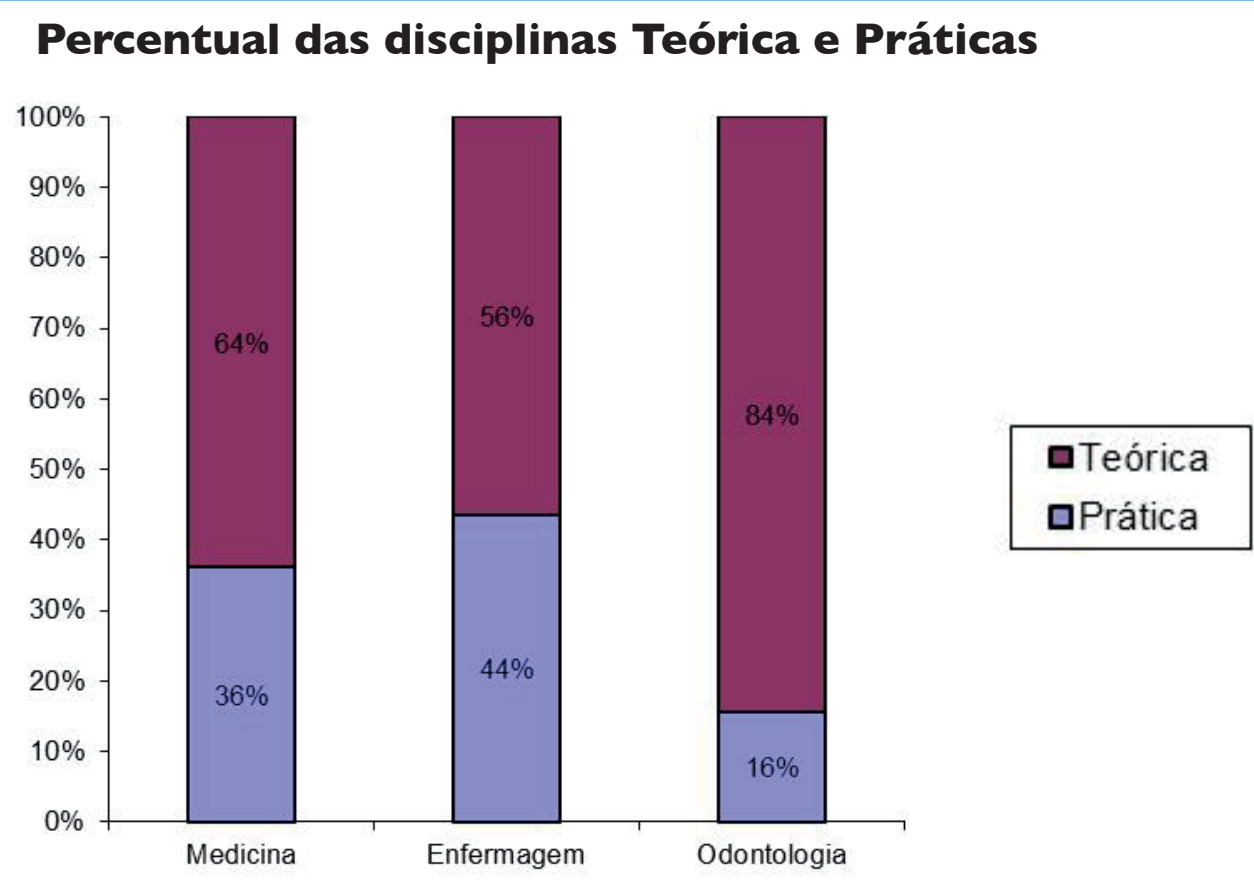

Gráfico 2 - Percentual das disciplinas obrigatórias Teóricas e Práticas que utilizam termos humanizantes ou correlatos.

Tabela I. As disciplinas com conteúdo prático maior que o teórico desenvolvem conceitos do homem e sua subjetividade, direcionando para o encontro com dois sujeitos individuais.

$\bigcirc$ curso de Enfermagem apresenta carga horária total de 4.940h, das quais somente I.700h em disciplinas que apresentaram os termos estudados. As disciplinas com carga horária teórica e prática compatíveis com o conceito de humanização estão apresentadas na Tabela 2. As demais disciplinas que desenvolvem o conceito humanista no curso são estritamente teóricas, priorizam o encontro dos sujeitos envolvidos - enfermeiro e paciente 
Tabela I - Disciplinas obrigatórias cujo conteúdo teórico e prático do curso de Medicina abordam o termo humanização ou correlatos.

\section{MEDICINA}

\begin{tabular}{|c|c|c|c|}
\hline Disciplina & Carga Horária & $\mathbf{T}$ & $\mathbf{P}$ \\
\hline Medicina Integral da Criança e do Adolescente I & 180 & 180 & 0 \\
\hline Medicina Integral do Adulto e do Idoso I & 180 & 180 & 0 \\
\hline Medicina Integral do Adulto e do Idoso II & 180 & 180 & 0 \\
\hline Psicologia Médica II & 30 & 30 & 0 \\
\hline Medicina Integral do Adulto e do Idoso III & 180 & 180 & 0 \\
\hline Medicina Integral da Criança e do Adolescente III & 180 & 180 & 0 \\
\hline Medicina Integral da Criança e do Adolescente IV & 180 & 180 & 0 \\
\hline Medicina Integral do Adulto e do Idoso IV & 180 & 180 & 0 \\
\hline Trabalho de Campo Supervisionado III & 420 & 0 & 420 \\
\hline Saúde Mental I & 30 & 30 & 0 \\
\hline Saúde e Sociedade II & 30 & 0 & 30 \\
\hline Trabalho de Campo Supervisionado II & 360 & 0 & 360 \\
\hline *Programa Prático Conceitual $3^{\mathrm{a}}$ e $4^{\mathrm{a}}$ fases & 1530 & 0 & 1530 \\
\hline Total & 3660 & 1320 & 2340 \\
\hline
\end{tabular}

- abordando o conceito de humanização e os direciona para situações específicas do processo "doença e cura". Essas disciplinas, com abordagem teórica, dão suporte para as disciplinas com carga prática.

No curso de Odontologia, 4.035h compõem a carga horária das disciplinas obrigatórias, e dessas, somente 1.220h apresentam o termo estudado em suas ementas e objetivos. Quatro disciplinas têm carga horária exclusivamente teórica, descrita na Tabela 3. Importante ressaltar que a disciplina de Fundamentos para a Clínica Odontológica é a única dentre as acima citadas que promove o despertar para um atendimento integral do paciente. As demais disciplinas apresentam conteúdo específico e voltado para a clínica odontológica. Elas detêm as maiores cargas horárias práticas e favorecem a permeabilidade de conhecimentos multi-disciplinares, desenvolvendo conhecimentos técnicos e científicos, com aplicabilidade de conceitos humanísticos na relação com os sujeitos (profissional-paciente-alunos).

\section{DISCUSSÃO}

Uma prática educativa humanizada na área da saúde coloca o homem como centro do processo de construção de cidadania, comprometida e integrada à realidade social e epidemiológica às políticas sociais e de saúde oportunizando a formação profissional contextualizada e transformadora (9).

As clínicas multidisciplinares e os estágios supervisionados se enquadram no grupo de disciplinas com conteúdo apenas prático, cujo conteúdo teórico foi ministrado anteriormente. Essas detêm as maiores cargas horárias práticas e favorecem a permeabilidade de conhecimentos multidisciplinares, desenvolvendo 
Tabela 2 - Disciplinas obrigatórias cujo conteúdo teórico e prático do curso de Enfermagem abordam o termo humanização ou correlatos.

ENFERMAGEM

\section{Disciplina}

Carga Horária

Ciências Humanas e Sociais 60

60

0

Enfermagem no Gerenciamento de Assistência à Saúde

90

30

60

Enfermagem na Saúde da Mulher I

90

30

60

Enfermagem na Saúde da Mulher II

90

30

60

Promoção de Saúde Mental

60

30

30

Enfermagem na Saúde da Criança e do Adolescente

100

20

80

Enfermagem na Saúde da Criança e do Adolescente II

120

30

90

Concepções, Saberes e Práticas do Cuidar em Saúde Mental

60

30

30

Relações de Trabalho em Saúde: Ética, Bioética e Legislação Profissional60

60

0

Fundamentos de Enfermagem I

160

70

90

Fundamentos de Enfermagem II

160

70

90

Enfermagem no Gerenciamento de Assistência à Saúde II

90

30

60

Ética

60

60

0

Enfermagem na Saúde do Adulto e do Idoso

160

40

120

Enfermagem em Unidades de Maior Complexidade

120

30

90

Didática

60

60

0

Pesquisa e Prática de Ensino II

100

0

100

Psicologia Aplicada à Saúde 60

Total

Carga Horária das Obrigatórias

1700

60 0

\section{Legenda Tabela 2 - T- teórico; P- prático}

4940

740

960

conhecimentos técnico-científicos com aplicabilidade de conceitos humanísticos na relação com os sujeitos (profissional-paciente-alunos).

As disciplinas cujas práticas são realizadas no laboratório da Universidade não são de atendimento direto ao paciente. Em um primeiro momento, elas desenvolvem a técnica específica de cada uma delas e preparam o aluno para a prática clínica.

Entende Sacristán (2000) que o conceito de currículo aceita diferentes significados, uma vez que, "além de ser susceptível a enfoques paradigmáticos diferentes, é utilizado para processos ou fases distintas do desenvolvimento curricular" (10). Fazendo uma crítica à visão tecnicista e classista de currículo, Apple (1982) concebe que: A área do currículo, mais do que outras áreas educacionais, tem sido dominada por uma perspectiva que poderia melhor chamar-se "tecnológica", na medida em que o principal interesse implica encontrar o melhor conjunto de meios para se alcançar objetivos educacionais pré-escolhidos. Para ele: [...] o currículo nunca é apenas um conjunto neutro de conhecimentos, 
Tabela 3 - Disciplinas obrigatórias cujo conteúdo teórico e prático do curso de Odontologia abordam o termo humanização ou correlatos.

\section{ODONTOLOGIA}

\begin{tabular}{|c|c|c|c|}
\hline Disciplina & Carga Horária & $\mathbf{T}$ & $\mathbf{P}$ \\
\hline Fundamentos para a Clínica Odontológica & 20 & 20 & 0 \\
\hline Saúde Bucal e Coletiva I & 60 & 30 & 30 \\
\hline Radiologia Odontológica & 80 & 20 & 60 \\
\hline Clínica Multidisciplinar I & 40 & 0 & 40 \\
\hline Clínica Multidisciplinar II & 100 & 0 & 100 \\
\hline Clínica Multidisciplinar III & 100 & 0 & 100 \\
\hline Clínica Multidisciplinar IV & 160 & 0 & 160 \\
\hline Endodontia & 120 & 40 & 80 \\
\hline Odontopediatria & 80 & 20 & 60 \\
\hline Odontogeriatria & 20 & 20 & 0 \\
\hline Estágio Supervisionado: Clínica Integrada Infantil & 80 & 0 & 80 \\
\hline Estágio Supervisionado: Clínica Integrada Geriátrica & 80 & 0 & 80 \\
\hline Odontologia Legal & 20 & 20 & 0 \\
\hline Estágio Supervisionado: Clínica Integrada do Adulto II & 160 & 0 & 160 \\
\hline Estágio Supervisionado: Saúde Coletiva II & 80 & 0 & 80 \\
\hline Orientação Profissional & 20 & 20 & 0 \\
\hline Total & 1220 & 190 & 1030 \\
\hline $\begin{array}{l}\text { Carga Horária das Obrigatórias } \\
\text { Legenda Tabela } 3 \text { - T- teórico; P- prático }\end{array}$ & 4035 & $16 \%$ & $84 \%$ \\
\hline
\end{tabular}

que de algum modo aparece nos textos e nas salas de aula de uma nação. Ele é sempre parte de uma tradição seletiva, resultado da seleção de alguém, da visão de algum grupo acerca do que seja conhecimento legítimo. É produto das tensões, conflitos e concessões culturais e econômicas que organizam e desorganizam um povo (I I).

A Medicina pode ser considerada a precursora dessa mudança de paradigma por ser o curso mais antigo na área da saúde, trazendo como necessidade deste novo cenário a integralidade da atenção, estendendo-se, portanto, às demais profissões da saúde. Observou-se que as disciplinas de Trabalho de Campo Supervisionado II e III, Saúde e Sociedade e Programa Prático Conceitual apresentam apenas conteúdo prático com uma carga horária de 2.340h, representando $64 \%$ do total da carga horária das disciplinas obrigatórias. Nessas disciplinas de conteúdo prático, ocorre o trabalho com as equipes multidisciplinares, colocando em prática o papel da extensão nas universidades. A Medicina Integral da Criança e Adolescente e a Medicina Integral do Adulto e do Idoso (I, II, III, IV) apresentam uma carga horária somente teórica, com $180 \mathrm{~h}$ em que se desenvolve o cuidar integral do indivíduo, pre- 
parando o aluno para complexidades práticas de forma crescente, compondo a definição da humanização, do adoecimento e prevenção em que ocorrerem os encontros entre sujeitos sociais (médico-paciente).

Envolver-se com a produção do cuidado em saúde remete ao campo da complexidade dos seus modos de gestão e das relações entre trabalhadores, gestores e usuários dos serviços de saúde. Nessa compreensão, a perspectiva ético-política nos processos de formação implica a inclusão dessa complexidade na interação entre sujeitos. Sem essa inter-relação não ocorre a sustentabilidade nos modelos de atenção e gestão no âmbito do SUS.

A construção de processos de formação em saúde, considerando esse referencial da "inclusão", implica estarmos atentos a essa complexidade e fazermos escolhas teóricometodológicas que expressem um campo de interlocução por entre os saberes, indissociadas de um método de formação. Essas escolhas são sempre escolhas ético-políticas ( I2).

No que concerne a todos os cursos analisados no presente estudo, embora as DCN sejam orientações a serem adotadas por todas as Instituições de Ensino Superior, a sua observância ainda não constitui a realidade da maioria dos cursos. A formação ainda se apresenta parcialmente pautada, principalmente, no exercício privado da profissão (13).

Difícil nos dias de hoje pensar em práticas profissionais que não estejam imediatamente comprometidas com o mundo, com o País em que vivemos, com as condições de vida da população brasileira, com o engajamento na produção de saúde que implique a produção de sujeitos autônomos, protagonistas, copartícipes e corresponsáveis por suas vidas. $\bigcirc$ currículo deve estar inserido em um contexto social, influenciar e ser influenciado por ele, principalmente por atitudes dos profissionais que por ele são formados (3).

Nosso estudo corrobora com Almeida e Chaves em 2009, (3) no que concerne ao estudo realizado sobre presença do termo humanização nas disciplinas do curso de Enfermagem na cidade de São Paulo. Dentre treze instituições de ensino superior, onze apresentaram maior percentual de disciplinas contendo o tema humanização ou correlato (de 54\% a $74 \%$ ), enquanto duas apresentaram 32\% e $43 \%$ de disciplinas contemplando o tema (3).

A proposta de humanização surge para combater a impessoalidade no atendimento de saúde e para tornar a relação profissional/paciente uma relação de afeto e respeito mútuo, sem abandonar a técnica necessária. $A$ humanização responde a tudo isso com princípios, diretrizes e dispositivos, todos acionados por um método (4). Assim, ela é uma aposta metodológica, uma maneira de lidar e intervir sobre problemas do cotidiano do SUS. Esse método é a tríplice inclusão: inclusão de pessoas, de coletivos e movimentos sociais e da perturbação, da tensão que essas inclusões produzem nas relações entre os sujeitos nos processos de gestão e de atenção, tomados como indissociáveis.

\section{CONCLUSÃO}

A identificação e análise do termo humanização na formação acadêmica em saúde dos cursos de Medicina, Enfermagem e Odontologia, de uma Instituição Pública de Ensino Superior, demonstrou a presença do termo nas ementas e/ou nos objetivos em 39\%, 34\% e $30 \%$ das disciplinas obrigatórias dos cursos de Medicina, Enfermagem e Odontologia, respectivamente, sendo predominante nas disciplinas teóricas. Com isso, foi possível perceber o empenho em contemplar as novas Diretrizes Curriculares tendo a humanização como elemento prioritário no que se refere à construção de novas relações entre alunos, docentes, usuários dos serviços e a rede SUS local.

\section{AGRADECIMENTOS}

Os autores agradecem todas as contribuições e sugestões realizadas pelo Prof. Dr. José Mauro Grangeiro.

Os autores declaram que não há conflito de interesse.

\section{Autora de correspondência:}

Larissa Maria Assad Cavalcante

Faculdade de Odontologia, Universidade

Federal Fluminense - UFF/RJ

Rua Mario Santos Braga, 28 - Centro, Niterói - RJ, 24020-140

lara_cvalcante@yahoo.com.br 


\section{REFERÊNCIAS BIBLIOGRÁFICAS}

I. Deslandes SF. Humanização: revisitando o conceito a partir das contribuições da sociologia médica. In: Deslandes SF, organizador. Humanização dos cuidados em saúde: conceitos, dilemas e práticas. Rio de Janeiro: Fiocruz; 2006. 33-47.

2. Rios IC. Caminhos da humanização na saúde: prática e reflexão. São Paulo: Áurea Editora; 2009.

3. Almeida DV, Chaves EC. $O$ ensino da humanização nos currículos de graduação em enfermagem. Einstein; 2009. 7(3):27|-8.

4. Brasil. Ministério da Educação e Cultura. Conselho Nacional de Educação/Câmara de Educação Superior. Resolução 3, de 7 de novembro de 200I. Institui Diretrizes Curriculares Nacionais do Curso de Graduação em Enfermagem. Brasilia: CNE/ CNS; 2001 .

5. Veiga PA. Projeto Político-Pedagógico da Escola: Uma Construção Possível. I a edição. Campinas: Papirus Editora; 1995.

6. Brasil. Ministério da Saúde. Secretaria-Executiva. Núcleo Técnico da Política Nacional de Humanização. Humaniza SUS: Política Nacional de Humanização: a humanização como eixo norteador das práticas de atenção e gestão em todas as ins- tâncias do SUS. Brasília: Ministério da Saúde; 2004.

7. Cellard A. A análise documental. Em: Poupart J, organizador. A Pesquisa qualitativa: enfoques epistemológicos e metodológicos. Petrópolis: Editora Vozes; 2008. I I-30.

8. Pimentel A. O método da análise documental: seu uso numa pesquisa historiográfica. Cad. Pesq; 200 I. I I 4( I): I 79- 95.

9. Moyses SJ. Políticas de saúde e formação de recursos humanos em Odontologia. Revista da ABENO; 2004. 4(I):30-7.

10. Sacristán JG. O Currículo, uma reflexão sobre a prática. Porto Alegre: Editora Artmed; 2000.

I I. Apple MW. Ideologia e Currículo. Em: Apple MW, organizador: A política do conhecimento oficial: faz sentido a idéia de um currículo nacional? São Paulo: Brasiliense; | 982. 59-7|. 12. Heckert ALC, Neves CABN. Modos de formar e modos de intervir: quando a formação se faz potência de produção de coletivo. In: PINHEIRO R.; MATTOS RA; BARROS MEB (Org.). Trabalho em equipe sob o eixo da integralidade: valores, saberes e práticas. Rio de Janeiro: Cepesc; 2007. I 45- 160. 13. Brasil. Ministério da Saúde. Ministério da Educação. A aderência dos cursos de graduação em Enfermagem, Medicina e Odontologia às Diretrizes Curriculares Nacionais. Brasília: Ministério da Saúde; 2006. 